Daten, empirische Graphen von Funktionen, mechanische Modelle realer Phänomene, ... )

Aber auch gut vorbereitete Lehrer können das Erreichen der genannten Ziele nicht erzwingen.

Die zu respektierende Tatsache, daß der Unterrichtsplanung Grenzen gesetzt sind, weil es in der Schule um Menschen geht, sollte aber gerade nicht dazu mißbraucht werden, die Forderung nach bestmöglicher Vorbereitung auf den Lehrerberuf, einen der wichtigsten und schwierigsten Berufe überhaupt, zu relativieren.

Adresse des Autors:

Prof. Dr. Heinrich Winter

Lehrstuhl für Didaktik der Mathematik

RWTH Aachen

52056 Aachen

\title{
Allgemeine Mathematik
}

\section{von Rudolf Wille}

Die Idee zu der Tagung „Allgemeine Mathematik - Mathematik für die Allgemeinheit“ hat sich im September 1994 auf einem Workshop über allgemeinbildenden Mathematikunterricht herausgebildet. Bei der Arbeit dieses Workshops wurde auf eindringliche Weise deutlich, daß die Diskussion über das, was für die Allgemeinheit heute Mathematik bedeutet, intensiviert werden muß. So entstand der Wunsch, sich im Rahmen einer Arbeitstagung allgemein mit dem derzeitigen Selbstverständnis der Mathematik, ihrem Verhältnis zur Welt sowie Fragen nach Sinn, Bedeutung und Zusammenhang mathematischen Tuns auseinanderzusetzen. Zwar liegen zu diesem Problemkreis schon vielfältig Arbeiten vor, doch fehlt es an Zusammenschau und fruchtbarer Diskurskultur, die eine differenzierte Reflexion allgemeiner Grundfragen der Mathematik präsent machen. Um die notwendige Breite der Auseinandersetzung zu garantieren, sollte die Tagung Wissenschaftler aus unterschiedlichen Bereichen wie vor allem Mathematik, Informatik, Fachdidaktik, Erziehungswissenschaft, Wissenschaftsphilosophie und -geschichte sowie Anwendungsgebieten der Mathematik zusammenführen.

Schon die Vorankündigung der Tagung wurde mit großem Interesse aufgenommen. So konnten die Veranstalter (H. W. Heymann, K. Radbruch, R. Wille) aus den genannten Wissenschaftsgebieten zwölf Hauptvortragende gewinnen, die in bezug auf das Anliegen der Tagung wichtige Themen behandelten. Zehn weitere Vorträge brachten für die Gesamtdiskussion des Tagungsthemas zusätzliche Anregungen. Über 100 Teilnehmer hörten die Vorträge und beteiligten sich engagiert an den Diskussionen. In diesem Beitrag soll versucht werden, Vorträge und Diskussionen in einer Zusammenschau wiederzugeben, was natürlich nur unvollständig geschehen kann. Zudem wird eine derartige Zusammenschau stets von Auffassung und Sichtweise des Berichterstatters geprägt. Ein Sammelband mit den wichtigsten Beiträgen zur Tagung wird einen vielfältigeren Einblick in den begonnenen Diskurs über das Tagungsthema geben.

"Mathematik für die Allgemeinheit" wurde in den Beiträgen und Diskussionen der Tagung als ein Thema gesehen, das das schwierige Verhältnis zwischen der akademischen Fachwissenschaft Mathematik als Expertenkultur und der alltäglichen Welt, in der die Menschen auf unterschiedliche Weise mit Mathematik umgehen und von ihr betroffen sind, ins Bewußtsein bringt. Daß es mit diesem Verhältnis nicht zum besten steht, machten besonders die Beiträge der Wissenschaftsjournalisten G. von Randow („Die Zeit“, Hamburg) und C. Pöppe („Spektrum der Wis- senschaft", Heidelberg) deutlich. Zwischen dem, was den akademischen Mathematiker bewegt, und dem, was allgemein als bedeutsam und interessant angesehen wird, klafft eine große Lücke, die nur schwer zu überbrücken ist. Diese Lücke besteht sogar zwischen der Mathematik in den Universitäten und der Mathematik in der Industrie, was H. Neunzert (Kaiserslautern) und A. Beutelspacher (Gießen) in ihren Beiträgen kritisch herausstellten. H. Neunzert sprach von den zwei Welten der Mathematik, die in ihrem Tun ganz unterschiedlichen Leitvorstellungen folgen: Der innermathematischen Vorstellung „guter" Theoriebildung steht die Forderung nach außermathematisch begründeter Bedeutung mathematischer Methoden und Theorien gegenüber. Auf der Tagung wurde auf vielfältige Weise diskutiert, wie dieser Gegensatz abgebaut werden kann. Positive Anregungen hierzu lieferte der Beitrag von A. Dress (Bielefeld) über die „Formensprache der Natur" als Gegenstand der Mathematik, in dem exemplarisch aufgezeigt wurde, wie mathematische Modelle bei der Beschreibung und Analyse neuartiger Phänomene erfolgreich eingesetzt werden können. Der grundlegenden Frage nach den Grenzen mathematischer Formalisierung ging W. Coy (Bremen) am Thema des „eigensinnigen“ Rechenautomaten nach. Er machte deutlich, wie das immer zu kurz greifende formale Denken von Metaphern geleitet war und ist, zunächst von der Metapher des Automaten, dann von der des 
Werkzeugs und neuerdings von der des Mediums; dabei entsteht der Sinn immer erst in Zusammenhang mit menschlichem Handeln, das die aktuellen Grenzen des Formalisierens bestimmt.

"Mathematik für die Allgemeinheit", was insbesondere die Verständigung über Mathematik beinhaltet, thematisiert auch das Verhältnis von akademischer Mathematik und schulischem Mathematikunterricht. In ihrem Beitrag zeigte L. HefendehlHebeker (Augsburg) an eindruckvollen Unterrichtsbeispielen, wie schwierig die Verständigung über Mathematik ist, wenn unterschiedliche Lebens- und Sinnvorstellungen nicht berücksichtigt werden. Hier ist es gerade der vielfach fehlende lebensweltliche Bezug akademischer Mathematik, der negative Auswirkungen auf den schulischen Mathematikunterricht hat. Nach L. Hefendehl-Hebeker gilt es vor allem, Offenheit und Bewußtheit zu erlangen über die Entwicklung, Bedeutung und Ziele mathematischer Denkinhalte. B. Andelfinger (Ulm), der einen „sanften Mathematikunterricht" propagiert, forderte in seinem Beitrag eine noch stärker lebensweltlich orientierte Unterrichtskultur, die davon geprägt ist, sich in einem Spannungsfeld von Weltdeutungen ernstzunehmen und aufzuklären. Für die akademische Mathematik bedeutet ein solcher Unterricht eine besondere Herausforderung, gilt es doch, das eigene Selbstverständnis in Hinblick auf mögliche Weltbezüge der Mathematik weitaus intensiver zu klären. H. W. Heymann (Bielefeld) stellte in seinem Beitrag die zentralen Thesen zum allgemeinbildenden Mathematikunterricht aus seiner Habilitationsschrift zur Diskussion. Ganz anders als in der später bundesweit aufgebrochenen Auseinandersetzung über die größtenteils mißverstandene These von der unmittelbaren Lebensvorbereitung beschäftigte die Tagungsteilnehmer vor allem, eine klareres Bild von der allgemeinen Bedeutsamkeit der unterschiedlichen mathematischen Lerninhalte und Qualifikationen zu erhalten, die von den sieben Thesen Heymanns angesprochen werden.

"Mathematik für die Allgemeinheit" weist als Thema auf die allgemeine Frage, welche Rolle der Fachwissenschaft Mathematik in unserer Welt zukommt. K. Radbruch (Kaiserslautern) behandelte in seinem Beitrag diese Frage aus wissenschaftsphilosophischer Sicht nach dem Zyklus von Kritik, Interpretation, Orientierung und Reflexion. Er votierte dabei für eine „Allgemeine Mathematik“ mit dem Ziel einer "Laisierung" mathematischen Wissens, durch die die Strukturen und Folgen dieses Wissens mit verständlichen Mitteln den begrifflich geschulten Laien und Wissenschaftlern anderer Disziplinen durchschaubar werden. M. Otte (Bielefeld) konzentrierte sich in seinem Beitrag auf die These, daß die Verbreitung mathematischer Konzepte und Vorstellungen untrenn- bar mit der Ausdehnung derselben auf neue Gegenstandsbereiche verbunden ist. Deshalb untersucht er diese Prozesse unter dem gemeinsamen Begriff der "Verallgemeinerung“. Als Wissenschaftshistoriker analysierte H. Mehrtens (Braunschweig) den innermathematischen Diskurs über das Selbstverständnis der Mathematik und ihr Verhältnis zur Welt. Als Schlußfolgerung stellte er heraus, daß nicht gefragt werden sollte „Was ist Mathematik?" sondern: „Wie wollen wir 'Mathematik' verstehen?" Wie R. Wille (Darmstadt) in seinem Beitrag zum Tagungsthema ausführte, sollte zum Selbstverständnis der Mathematik gehören, daß die Mathematik und jede ihrer Teildisziplinen an „Allgemeiner Mathematik“ teilhaben; dabei wird "Allgemeine Mathematik" als die Gesamtheit aller Bemühungen verstanden, Mathematik offenzulegen und zugänglich zu machen, damit sich die Allgemeinheit mit den Inhalten, Folgen und Auswirkungen mathematischen Tuns produktiv und kritisch auseinandersetzen kann.

Die Vorstellungen einer Allgemeinen Mathematik haben sich aus der langjährigen Beschäftigung mit Hartmut von Hentigs Forderung nach Restrukturierung der Wissenschaften entwickelt, die er in seinem Buch „Magier oder Magister? Über die Einheit der Wissenschaft im Verständigungsprozeß" herausgearbeitet hat. Deshalb war auf der Tagung eine Extradiskussion zum Thema der „Restrukturierung von Mathematik" angesetzt. In einem längeren Eingangsreferat erläuterte $\mathrm{H}$. von Hentig (Berlin), wie er zur Forderung nach Restrukturierung der Wissenschaften gekommen ist, was für ihn u. a. heißt, daß die Disziplinen ihre Disziplinarität überprüfen müssen. Die Restrukturierung der Wissenschaften in sich - um sie besser lernbar, gegenseitig verfügbar und allgemeiner (d. h. auch jenseits der Fachkompetenz) kritisierbar zu machen - kann und muß nach Mustern vorgenommen werden, die den allgemeinen Wahrnehmungs-, Denk- und Handlungsformen unserer Zivilisation entnommen sind. In der anschließenden Diskussion wurden zahlreiche Aspekte der Restrukturierung von Mathematik angesprochen und mit Bezug auf das Tagungsthema diskutiert.

Insgesamt hat die Tagung klar werden lassen, daß es einer allgemeinen Diskurskultur bedarf, die eine breite Auseinandersetzung über die Mathematik und ihre Bedeutung für die Allgemeinheit ermöglicht. Deshalb soll der begonnene Diskurs auf einer weiteren Tagung zum Thema „Allgemeine Mathematik: Ordnen, Strukturieren, Mathematisieren" (7.-9. Oktober 1996 an der TH Darmstadt) fortgesetzt werden.

Adresse des Autors:

Prof. Dr. Rudolf Wille

TH Darmstadt, Fachbereich Mathematik

Schloßgartenstr. 7, 64289 Darmstadt 